https://helda.helsinki.fi

\title{
TIA model is attainable in Wistar rats by intraluminal occlusion of the MCA for 10 min or shorter
}

\section{Tolvanen, Durukan}

2017-05-15

Tolvanen, D , Tatlisumak , E , Pedrono , E , Abo-Ramadan , U \& Tatlisumak , T 2017 , ' TIA model is attainable in Wistar rats by intraluminal occlusion of the MCA for 10 min or shorter ' , Brain Research , vol. 1663 , pp. 166-173 . https://doi.org/10.1016/j.brainres.2017.03.010

http://hdl.handle.net/10138/235565

https://doi.org/10.1016/j.brainres.2017.03.010

unspecified

publishedVersion

Downloaded from Helda, University of Helsinki institutional repository.

This is an electronic reprint of the original article.

This reprint may differ from the original in pagination and typographic detail.

Please cite the original version. 
Research report

\title{
TIA model is attainable in Wistar rats by intraluminal occlusion of the MCA for 10 min or shorter
}

\author{
A. Durukan Tolvanen ${ }^{\mathrm{a}, \mathrm{b}}$, E. Tatlisumak ${ }^{\mathrm{c}, *}$, E. Pedrono ${ }^{\mathrm{a}}$, U. Abo-Ramadan ${ }^{\mathrm{a}}$, T. Tatlisumak ${ }^{\mathrm{a}, \mathrm{b}, \mathrm{d}}$ \\ ${ }^{a}$ Experimental MRI Laboratory, Biomedicum Helsinki, Helsinki, Finland \\ ${ }^{\mathrm{b}}$ Department of Neurology, Helsinki University Central Hospital, Helsinki, Finland \\ ${ }^{\mathrm{c}}$ Department of Anatomy, Manisa Celal Bayar University, Faculty of Medicine, Manisa, Turkey

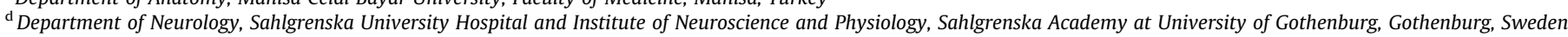

\section{A R T I C L E I N F O}

\section{Article history:}

Received 13 December 2016

Received in revised form 15 February 2017

Accepted 6 March 2017

Available online 11 March 2017

\section{Keywords:}

Animal model

Cerebral ischemia

Magnetic resonance imaging

Middle cerebral artery

Rat

Transient ischemic attack

\begin{abstract}
A B S T R A C T
Transient ischemic attack (TIA) has received only little attention in the experimental research field. Recently, we introduced a TIA model for mice, and here we set similar principles for simulating this human condition in Wistar rats. In the model: 1) transient nature of the event is ensured, and 2) $24 \mathrm{~h}$ after the event animals are free from any sensorimotor deficit and from any detectable lesion by magnetic resonance imaging (MRI). Animals experienced varying durations of ischemia $(5,10,12.5,15,25$, and $30 \mathrm{~min}, \mathrm{n}=6-8$ per group) by intraluminal middle cerebral artery occlusion (MCAO). Ischemia severity and reperfusion rates were controlled by cerebral blood flow measurements. Sensorimotor neurological evaluations and MRI at $24 \mathrm{~h}$ differentiated between TIA and ischemic stroke. Hematoxylin and eosin staining and apoptotic cell counts revealed pathological correlates of the event. We found that already $12.5 \mathrm{~min}$ of ischemia was long enough to induce ischemic stroke in Wistar rats. Ten min or shorter durations induced neither gross neurological deficits nor infarcts visible on MRI, but histologically caused selective neuronal necrosis. A separate group of animals with $10 \mathrm{~min}$ of ischemia followed up to 1 week after reperfusion remained free of infarction and any MRI signal change. Thus, 10 min or shorter focal cerebral ischemia induced by intraluminal MCAO in Wistar rats provides a clinically relevant TIA the rat. This model is useful for studying molecular correlates of TIA.
\end{abstract}

(c) 2017 Elsevier B.V. All rights reserved.

\section{Introduction}

Transient ischemic attack (TIA), according to its new tissuebased definition, is a brief episode of neurological dysfunction due to a vascular cause, with no detectable infarction (Albers et al., 2002; Easton et al., 2009). To differentiate between TIA and stroke, it is necessary to utilize brain imaging with MRI including diffusion-weighted scans (Easton et al., 2009). This new concept of TIA has inspired us to develop a TIA model for mice (Pedrono

Abbreviations: CBF, cerebral blood flow; DWI, diffusion-weighted imaging; HE, hematoxylin and eosin; LDF, laser Doppler flowmetry; MCAO, intraluminal suture occlusion of the middle cerebral artery; MRI, magnetic resonance imaging; TIA, transient ischemic attack; TTC, 2,3,5,-triphenyltetrazolium chloride; TUNEL, terminal deoxynucleotidyl transferase-mediated dUTPbiotin nick end-labeling.

* Corresponding author.

E-mail addresses: Aysan.Durukan-Tolvanen@hus.fi (A. Durukan Tolvanen), ertugrul.tatlisumak@gmail.com (E.Tatlisumak),Eric@ericpedrono.com (E.Pedrono), Aboramadan@hotmail.com (U. Abo-Ramadan), Turgut.Tatlisumak@neuro.gu.se (T. Tatlisumak). et al., 2010) and rats, convenient species to use in experimental focal brain ischemia research.

Intraluminal suture occlusion of the middle cerebral artery (MCAO) is a widely used method in experimental stroke. Its major advantage is the absolute control on the duration of ischemia (reperfusion is abrupt by the time of suture withdrawal) and on the rate of reperfusion (possibility for complete recanalization, unless a clot is formed), simulating ischemia-reperfusion of a large cerebral artery in humans. The relevance of the suture occlusion model should be better appreciated because of the recently opened era of thrombectomy (Tatlisumak, 2015).

Brief periods of focal cerebral ischemia are generally used in rats as a preconditioning event. For this purpose, generally 2 to 10 min-ischemia is applied with the intraluminal MCAO method (Alkayed et al., 2002; Puisieux et al., 2004; Glantz et al., 2005; Naylor et al., 2005), but as long as 20 (Glazier et al., 1994) to 30 min- ischemia (Yoshida et al., 2004) was used. In Wistar rats, ischemia duration longer than 3 min seems sufficient to induce injury (Puisieux et al., 2004), but not an infarction visible with 
2,3,5-triphenyltetrazolium chloride (TTC) staining (Alkayed et al., 2002). 10 min of ischemia induced no detectable infarct with cresyl violet staining in spontaneously hypertensive rats (Naylor et al., 2005), but selective neuronal necrosis in Sprague-Dawley rats (Li et al., 2000). In this strain, three times of $10 \mathrm{~min}$ ischemia with 45 min intervals caused no lesion at $24 \mathrm{~h}$ but at $72 \mathrm{~h}$ infarction was noticed (Chen et al., 1996). These studies showed us that outcome of a brief episode of ischemia varies depending on animal strain used, the method of lesion evaluation, and the time-point of outcome evaluation.

Both mice and rats are the most commonly used animals in focal brain ischemia research. As we described a TIA model for mice previously (Pedrono et al., 2010), now we decided to validate the same model in Wistar rats as ischemia thresholds and responses to ischemic insults of equal severity may differ between species and the results of mice studies cannot directly apply to rat studies. Here, we aimed to develop a TIA model in male Wistar rats, by systematic application of increasing durations of transient focal cerebral ischemia. To achieve a close clinical relevance to TIA (Easton et al., 2009), in our model we used suture occlusion model of the MCAO, assessed the outcome with sensorimotor testing and MRI at $24 \mathrm{~h}$ after reperfusion. After detecting the critical ischemia duration limiting the outcome to TIA rather than stroke, we tested the presence of delayed infarction with this duration.

\section{Results}

\subsection{Physiological parameters (Table)}

Rectal temperatures were steady and within physiological ranges during the entire experiment $(P>0.05$ for each group). Baseline body weights varied from 250 to $450 \mathrm{~g}(P<0.001$, control group was relatively lighter and 10-min group heavier). At $24 \mathrm{~h}$, weight loss was around $13 \%$, as compared with baseline weight $(P<0.001)$ with no intergroup difference $(P=0.111)$. Seven-day group's weight follow-up showed no changes over time $(P=0.135)$.

\section{2. $\mathbf{C B F}$}

MCAO caused an immediate CBF drop of $76 \pm 9 \%$ from the baseline level, which was similar for all ischemia groups $(P=0.45)$ (Fig. 1, Table 1). CBF values recovered to $74 \pm 21 \%$ of baseline 30 min after reperfusion. This was statistically different between groups $(P=0.013)$. However, this difference has vanished at $24 \mathrm{~h}$ $(P=0.285)$, when $\mathrm{CBF}$ values became approximately equal to those of baseline $(P=0.946)$.

\subsection{Neurological deficits and infarcts}

Fig. 2A resumes typical MRI findings of the study. MRI lesions in the 12.5- ( 1 in 6 animals, with lesion volume of $27 \mathrm{~mm}^{3}$ ) and 15min groups ( 3 in 7 animals) were all limited to lateral caudaputamen. No animals in the 5- or 10-min ischemia groups including the 10-min ischemia long-term follow-up group as well as no animals in the sham (control) group showed any ischemic changes or other abnormalities in MRI. In the 25-min group, all animals developed infarction in the caudaputamen, of these 3 showed also patchy cortical involvement. In the 30-min group, subcortical involvement was again a sustained finding, with cortical involvement in half of the rats. Extended reperfusion period of one week in a separate group of animals subjected to 10-min ischemia (7-day group), disclosed no further ischemic damage neither at $72 \mathrm{~h}$ nor at 7 days on MR images. MRI-based infarct volumes are shown in Fig. 2B. Neurological deficit findings and lesion volumes are resumed in Table 1. In the 1-week follow-up group of animals with 10-min of MCAo, total brain volumes were measured from the MRI at Day 1 were

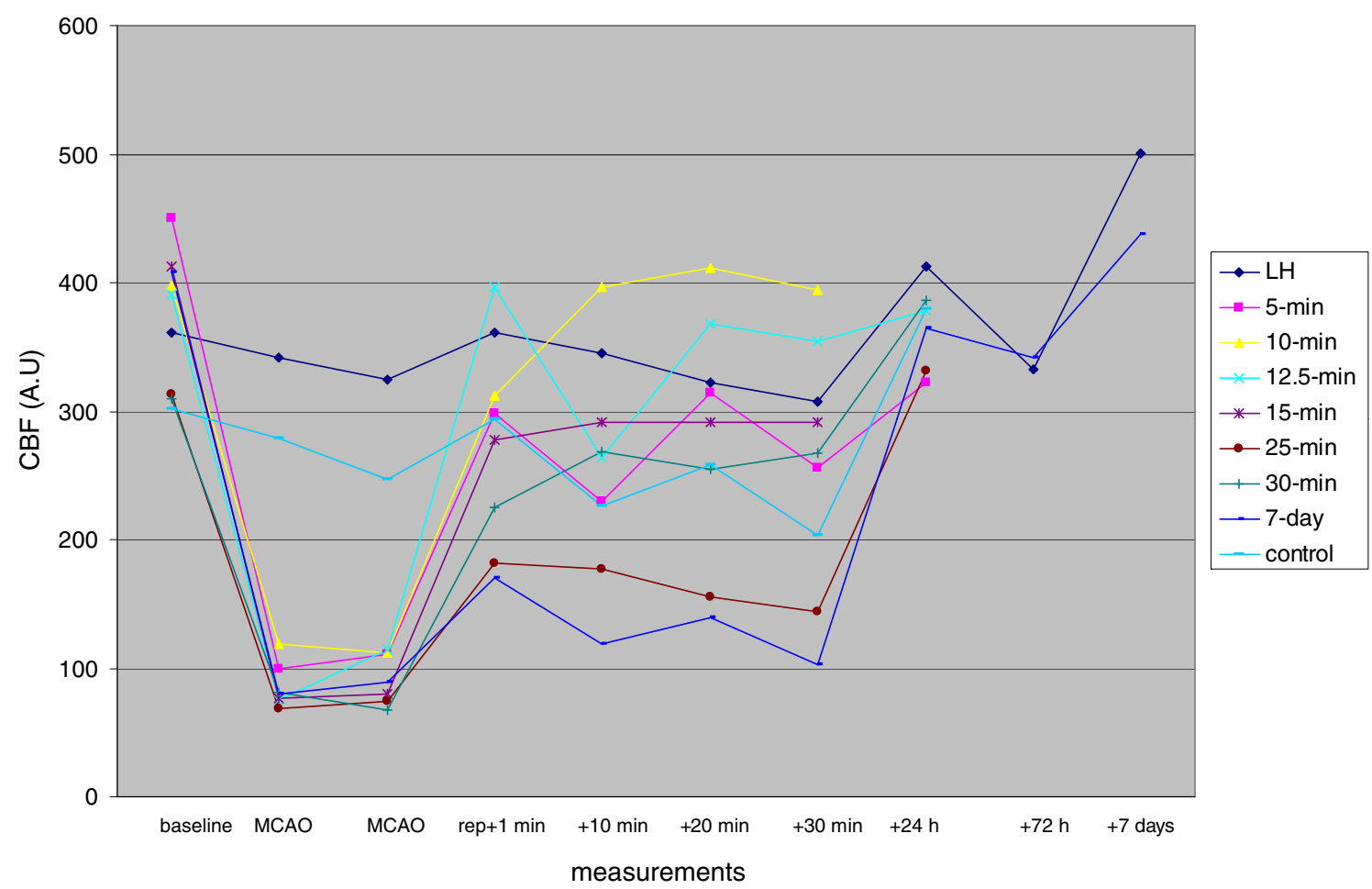

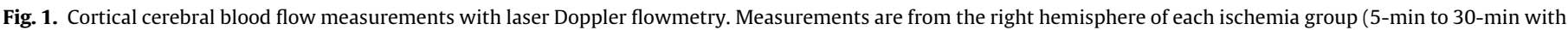

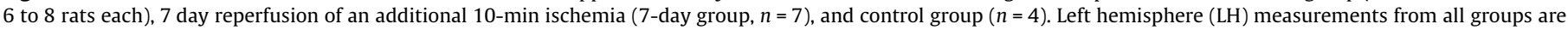
averaged. 
Table 1

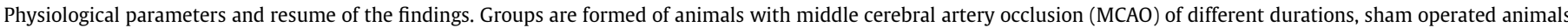

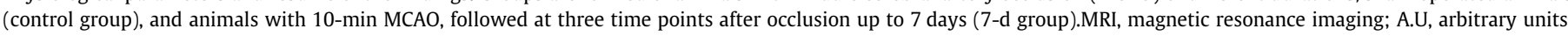

\begin{tabular}{|c|c|c|c|c|c|c|c|c|c|c|c|}
\hline & \multicolumn{2}{|l|}{ Groups $(n)$} & \multirow{2}{*}{$\begin{array}{l}12.5-\mathrm{min} \\
(6)\end{array}$} & \multirow{2}{*}{$\begin{array}{l}\text { 15-min } \\
(7)\end{array}$} & \multirow{2}{*}{$\begin{array}{l}25-\min \\
(7)\end{array}$} & \multirow{2}{*}{$\begin{array}{l}30-\mathrm{min} \\
(6)\end{array}$} & \multirow{2}{*}{$\begin{array}{l}\text { Control } \\
(4)\end{array}$} & \multicolumn{4}{|l|}{$7-d(7)$} \\
\hline & $\begin{array}{l}5-\min \\
(8)\end{array}$ & $\begin{array}{l}10-\mathrm{min} \\
(6)\end{array}$ & & & & & & MCAO-day & $24 \mathrm{~h}$ & $72 \mathrm{~h}$ & $7 \mathrm{~d}$ \\
\hline Body weights (g) baseline & $313 \pm 40$ & $403 \pm 49$ & $323 \pm 12$ & $365 \pm 55$ & $327 \pm 30$ & $279 \pm 16$ & $257 \pm 3$ & $271 \pm 20$ & & $259 \pm 21$ & $277 \pm 18$ \\
\hline reperfusion $+24 \mathrm{~h}$ & $296 \pm 30$ & & $309 \pm 12$ & $271 \pm 33$ & $300 \pm 28$ & $263 \pm 15$ & $235 \pm 8$ & & $259 \pm 26$ & & \\
\hline Temperatures $\left({ }^{\circ} \mathrm{C}\right)$ baseline & $37 \pm 0.3$ & $37 \pm 0.3$ & $37 \pm 0.5$ & $37 \pm 1$ & $37 \pm 0.6$ & $37 \pm 0.6$ & $37 \pm 0.6$ & $37 \pm 0.4$ & & & \\
\hline MCAO & $37 \pm 0.8$ & $37 \pm 0.6$ & $37 \pm 0.6$ & $37 \pm 0.7$ & $37 \pm 0.6$ & $37 \pm 1.3$ & $37 \pm 0.7$ & $37 \pm 0.5$ & & & \\
\hline reperfusion + $30 \mathrm{~min}$ & $37 \pm 1$ & $37 \pm 0.3$ & $37 \pm 2$ & $37 \pm 1.2$ & $37 \pm 0.6$ & $37 \pm 0.8$ & $37 \pm 0.5$ & $36 \pm 1.3$ & & & \\
\hline C.B.F. (A.U.) baseline & $449 \pm 195$ & $397 \pm 89$ & $391 \pm 91$ & $412 \pm 80$ & $314 \pm 148$ & $310 \pm 88$ & $302 \pm 112$ & $408 \pm 203$ & & & \\
\hline MCAO & $99 \pm 44$ & $119 \pm 39$ & $76 \pm 35$ & $76 \pm 49$ & $69 \pm 35$ & $81 \pm 38$ & $279 \pm 67$ & $80 \pm 22$ & & & \\
\hline reperfusion $+30 \mathrm{~min}$ & $256 \pm 148$ & $395 \pm 110$ & $355 \pm 96$ & $292 \pm 105$ & $144 \pm 68$ & $268 \pm 165$ & $203 \pm 79$ & $102 \pm 39$ & & & \\
\hline reperfusion + $24 \mathrm{~h}$ & $322 \pm 86$ & no & $379 \pm 90$ & no & $331 \pm 113$ & $387 \pm 44$ & $379 \pm 184$ & & $365 \pm 89$ & & \\
\hline Sensorimotor deficit $(n)$ & $0(0 \%)$ & $0(0 \%)$ & $0(0 \%)$ & $2(29 \%)$ & $2(29 \%)$ & $4(67 \%)$ & $0(0 \%)$ & & $0(0 \%)$ & $0(0 \%)$ & $0(0 \%)$ \\
\hline MRI lesion ( $n,(\%))$ & $0(0 \%)$ & $0(0 \%)$ & $1(17 \%)$ & $3(43 \%)$ & $7(100 \%)$ & $6(100 \%)$ & $0(0 \%)$ & & $0(0 \%)$ & $0(0 \%)$ & $0(0 \%)$ \\
\hline Lesion volume in MRI $\left(\mathrm{mm}^{3}\right)$ & 0 & 0 & 27 & $43 \pm 16$ & $70 \pm 49$ & $113 \pm 92$ & 0 & & 0 & 0 & 0 \\
\hline
\end{tabular}
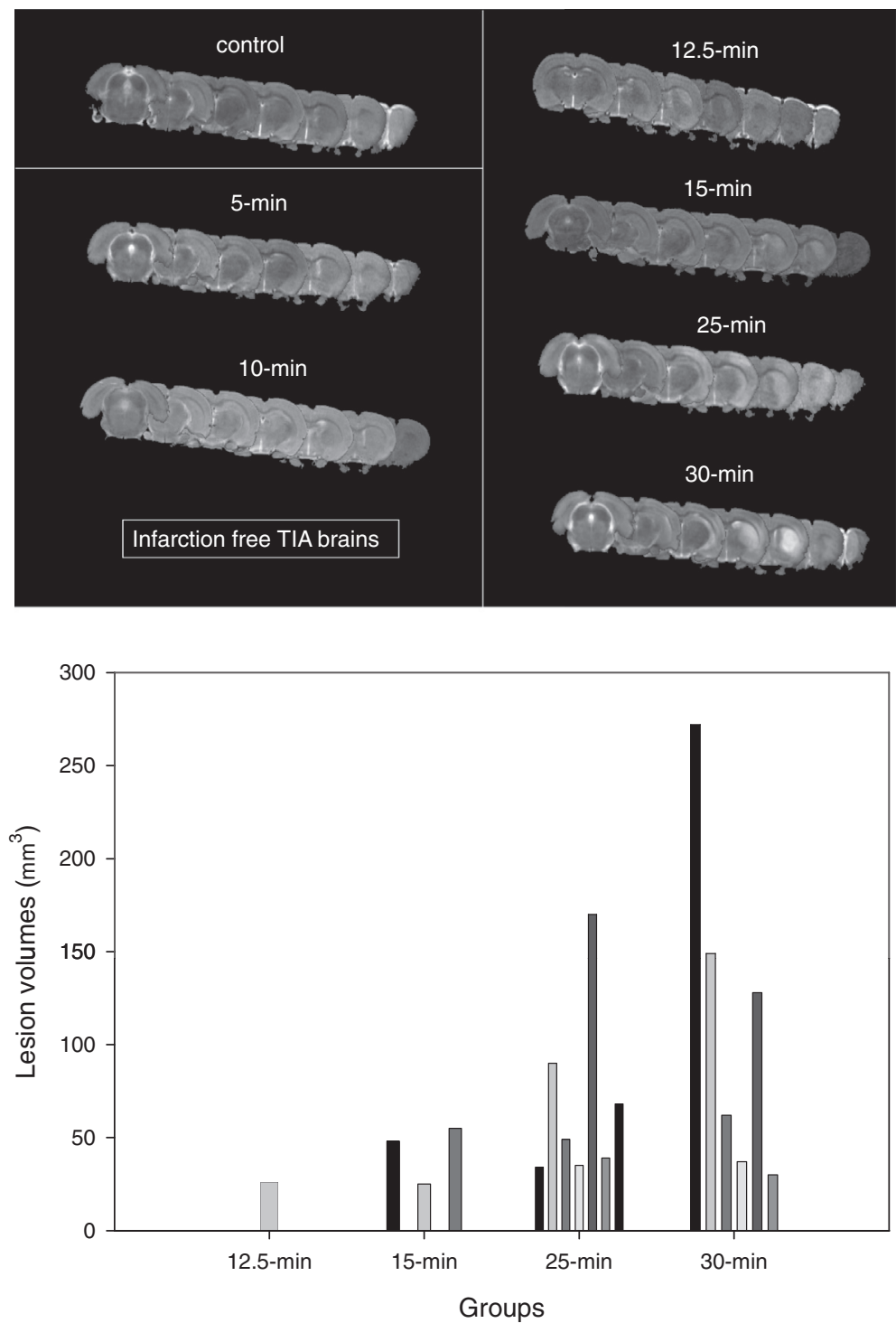

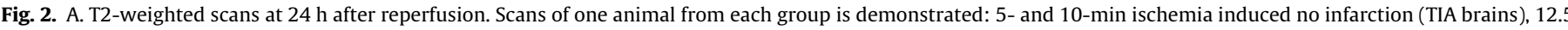

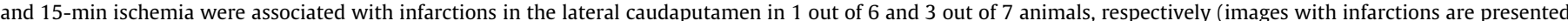

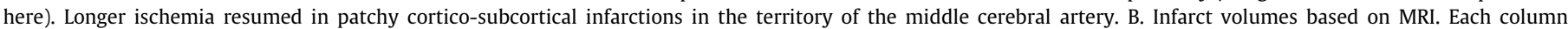
represents infarct volume of an individual animal. 
in average 1652.14 cubic millimeters and at Day 7 were 1652.80 cubic millimeters giving a $\mathrm{P}$ value of 0.965 ( $\mathrm{Cl}$ at $95 \%$ is -35.56 to 34.24$)$.

\subsection{HE results}

In line with previous data, the longer the duration of ischemia, the more pronounced the ischemic damage was $(R=0.762$, $P<0.001$, Fig. 3A). Transient ischemia of $5-$ and 10 -min resulted

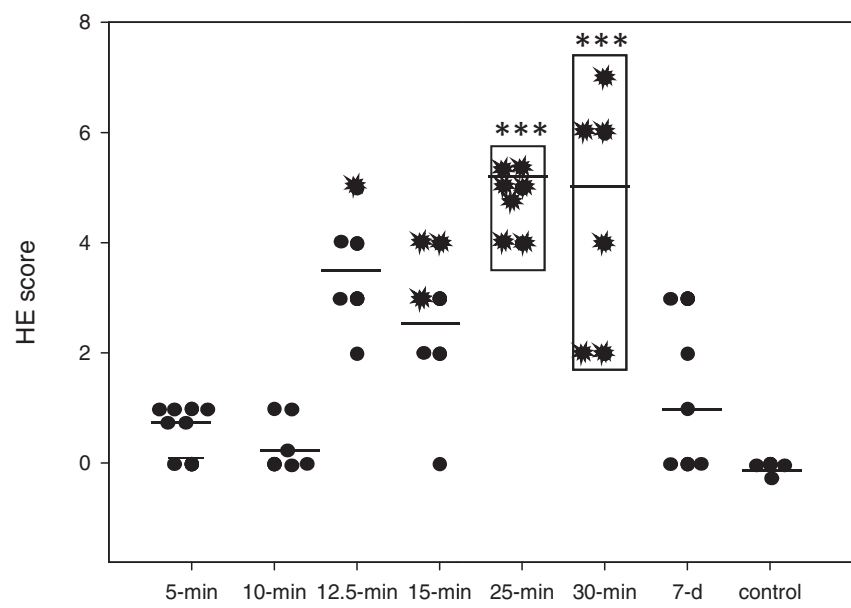

Groups

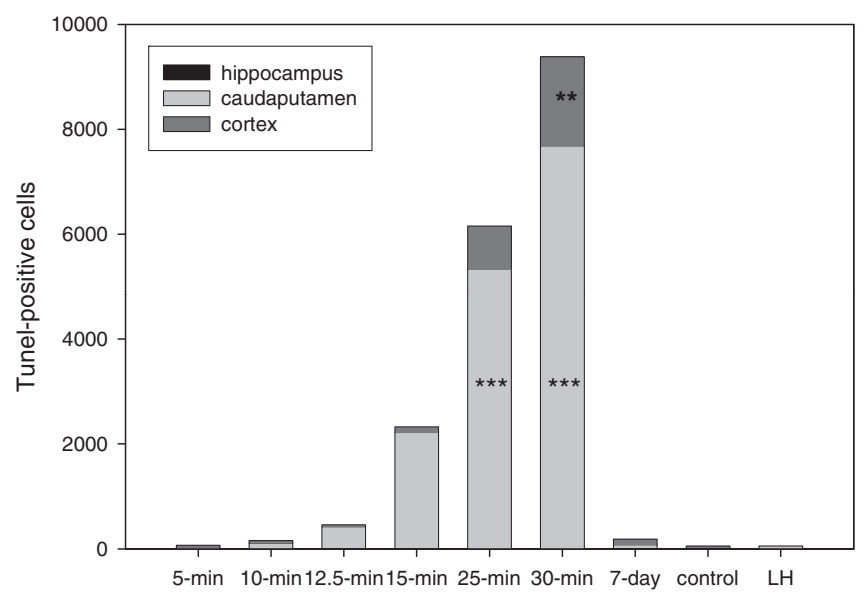

Groups

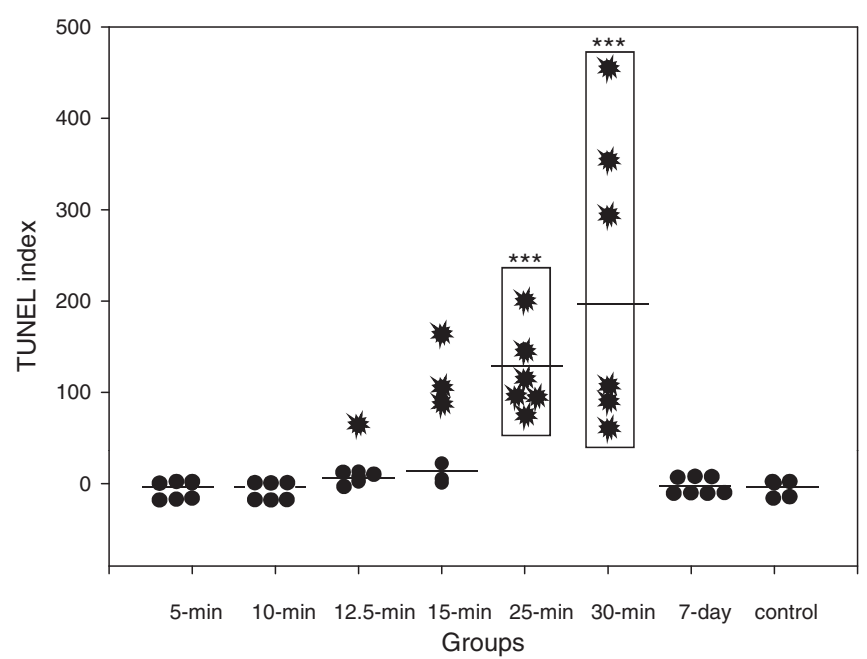

in scattered ischemic neuronal changes (hypereosinophilic neurons and shrunken neurons), mainly in hippocampus and in a lesser extent in the caudaputaminal regions of the right (ischemic) hemisphere in all animals. The outcome after 12.5-min ischemia was variable: subcortical selective neuronal necrosis was a feature in 2 of 6 animals and 1 of 6 showed small infarction.15-min or longer durations induced either selective neuronal necrosis or infarction. HE scores were different only in 25-min and 30-min ischemia groups (vs control, $P<0.001$ ). Between ischemia groups, HE scores in 5-min and 10-min groups differed from those of 25min and 30 -min groups $(P<0.001)$. Seven days after 10 -min ischemia no additional ischemic damage was observed $(P=0.125)$.

\subsection{TUNEL}

Left (intact) hemispheric TUNEL-positive cell counts were similar among groups $(P=0.537)$. Right hemispheric counts of TUNELpositive cells were significantly higher as compared with left hemispheric counts $(P<0.001$, Fig. 3B) and 25 - and 30 -min ischemia groups had a higher TUNEL index than had control group and 5and 10 -min groups $(P<0.001)$ (Fig. 3C). In regional TUNEL analysis (Fig. 3B), we observed apoptotic cells mostly in the caudaputamen of 25- and 30-min groups, number of cortical apoptotic cells reached statistical significance with 30-min-duration of ischemia. Hippocampus showed no significant apoptosis in any group $(P=0.748)$. Number of apoptotic cells induced by 10 -min ischemia were not increased at 1 week, as compared with those at $24 \mathrm{~h}$ $(P=0.366)$. Severity of apoptosis and ischemia duration were in good correlation $(R=0.78, P<0.001)$.

\section{Discussion}

A new definition of TIA was proposed in 2009 and received widespread acceptance (Easton et al., 2009). This tissue-based TIA definition relies on the exclusion of infarction with imaging methods, preferably with MRI. Although neurological or imaging correlates of TIA are absent in the short term, thereafter a cognitive decline has been reported (Takahashi et al., 2009). In addition to heralding forthcoming ischemic strokes, TIAs are associated with global brain atrophy (Walters et al., 2003). Repeated TIAs are associated with cognitive decline (Bakker et al., 2003; Takahashi et al., 2009) suggesting that TIA likely leads to some degree of permanent brain injury that is beyond the detection limits of MRI. Because histopathologic studies of patients with TIA are not feasible, an appropriate animal model of TIA may facilitate analyses of changes within the neurovascular unit and the molecular determinants of tissue fate after TIA, and it would assist in identifying cellular

Fig. 3. 5- to 30 -min ischemia was induced $(n=6-8)$, reperfusion lasted $24 \mathrm{~h}$, except 7 days in the 7-day group with 10 -min ischemia $(n=7)$. Control group consisted of 4 sham-operated animals. A. Impact of ischemia as hematoxylin and eosin (HE) scores. Ischemic changes in the hippocampus, caudaputamen, and frontoparietal cortex of both hemispheres were scored $(0=$ no change, $1=$ scattered neuronal changes, $2=$ selective neuronal necrosis, and $3=$ infarction) and summed. HE scores differed only in 25-min and 30-min groups (vs control ${ }^{* * *}, P<0.001$ ). Between ischemia groups, HE scores of 5-min and 10-min groups were different from those of 25- and 30 min groups $(P<0.001)$. Animals with infarct are presented with beams extending from a black circle. B. Terminal deoxynucleotidyl transferase-mediated dUTPbiotin nick end-labeling (TUNEL). Regional TUNEL-positive cell counts of the ipsilateral (right) hemisphere. For comparison averaged left hemisphere counts from all groups is also shown. 25- and 30-min ischemia induced significant apoptosis in the caudaputamen $\left({ }^{* * *}, P<0.001\right)$ and 30 -min also in the cortex $\left({ }^{* *}, P=0.003\right)$. Hippocampal counts were similar among groups $(P=0.748) .10 \mathrm{~min}$-ischemia caused no further apoptosis at the end of 7 days follow up (10-min vs 7-day group, $P=0.445)$. C. TUNEL index as the total right hemisphere counts divided by the left hemisphere counts. Groups of 25- and 30-min ischemia have higher index than have the control group, 5-min, and 10-min ischemia groups $\left({ }^{* * *}, P<0.001\right)$. Animals with infarct are presented with beams extending from a black circle. 
and molecular substrates of mild ischemic injury in a spatial pattern and with a dose-response gradient corresponding to the increasing severity of ischemic insult. Furthermore, such a model may aid the discovery of therapies targeting the adverse consequences of TIA, such as necrotic and apoptotic injury cascades, and promoting neuroprotection. Future rodent studies utilizing this TIA model and incorporating long-term follow-up together with sophisticated neurological, behavioral, imaging, and postmortem examinations may fill this gap of knowledge while this present study offers the necessary tools to these forthcoming studies.

There exist only few data on TIA in the experimental literature. Appreciating the need for an optimized animal model for TIA, we previously described such a model in mice (Pedrono et al., 2010). The novelty of the model was neither the focal ischemia technique used (in contrary, we utilized the most common method, the intraluminal suture occlusion of the MCA) nor any of the techniques used for outcome evaluation (a simple sensorimotor testing and $\mathrm{MRI}$ ), but was the implementation of 3 criteria when testing varying durations of MCAO. Due to these criteria the mouse TIA model achieved a clinical relevance, that we could identify suitable durations of MCAO leading to neurologically (grossly) intact and MRIlesion free TIA events in mice. Here, we used a similar approach for developing an equally relevant TIA model in rats. Outcomes of 5 to $30 \mathrm{~min}$ of focal ischemia were evaluated in male Wistar rats to describe a TIA model. Predetermined criteria of TIA model were: 1) LDF should indicate a successful occlusion and reperfusion, 2) no sensorimotor deficit should be observed at $24 \mathrm{~h}$ after reperfusion, and 3 ) at this time-point no lesion on magnetic resonance images should be found. The necessity of the first criterion raises from the fact that intraluminal MCAO model may fail for different reasons and, not rarely, inadequate occlusion and reperfusion occurs (Schmid-Elsaesser et al., 1998). These handicaps can easily be detected by LDF, monitoring cerebral blood flow through intact skull. If one aims to control the severity of ischemia, the concomitant use of LDF during intraluminal MCAO is indispensable. This holds true especially in brief ischemia applications. Second criterion relies on sensorimotor evaluation of animals. A number of sensorimotor and behavioral tests serve to investigate the impact of focal cerebral ischemia (Hunter et al., 2000). Behavioral tests are particularly important in drug studies. However, these were developed mostly for mice. Furthermore, TIA's clinical diagnosis is based often on patients' description of the symptoms, which are frequently sensorimotor. For this reason, when studying a TIA-like episode, rather than sophisticated behavioral tests neurological evaluation of sensorimotor functions are preferable. Third criterion introduces the use of MRI, which was proposed as the diagnostic imaging method in patients presenting with TIA (Easton et al., 2009). In the present study, these three criteria were fulfilled for $10 \mathrm{~min}$ or shorter MCAO, proving a suitable TIA model for Wistar rats. A transition between TIA and infarction occurs between 12.5 and $15 \mathrm{~min}$ of MCAo. Longer MCAO durations were associated with infarctions. Infarction threshold with intraluminal MCAO model was similar in NMRI mouse (Pedrono et al., 2010). Spontaneously hypertensive rats (Ejaz et al., 2015) and Swiss albino mice were devoid of infarction after $15 \mathrm{~min}$ of surgically induced distal MCAO (Arsava et al., 2009). In TIA patients, $30 \mathrm{~min}$ seemed like a cut off duration which differentiates between MRI negative and positive outcomes (Inatomi et al., 2004).

Higher species have probably less vulnerability to ischemia than rodents do. Moreover, strain and vendor differences may influence sensitivity to ischemia (Oliff et al., 1995). Future studies in phylogenetically higher animals (such as cats, dogs, pigs, and primates) searching for ischemic thresholds will allow for interspecies comparisons as well as form a base for investigating a number of phenomena such as why different species may be having differing thresholds. A number of factors such as gray versus white matter proportions, neuron density, formation of the Willis circle, collateral circulation, cerebral blood flow values, age, and gyrencephalic versus lissencephalic brain as well as yet unknown intrinsic factors may play important roles. Accordingly, the application of our TIA model to any rat strain may require preliminary MCAO experiments with 10 and 12.5 min duration to determine infarction threshold specific to that strain. Ejaz et al. (2015) developed a TIA model for spontaneously hypertensive rats. This model includes 15-min microclip occlusion of distal MCA leaving animals free from infarction and gross neurological deficits. Interestingly, a more sophisticated sensorimotor test (sticky label test) was sensitive to show deficits, of which pathological correlates were selective neuronal necrosis. In rats as brief as $8 \mathrm{~min}$ of focal ischemia resumes in selective neuronal necrosis (Li et al., 1999). As well apoptosis is induced after brief ischemia (Fink et al., 1998; Lee et al., 2002). In our study, 25 and 30 min of MCAO, but not milder ischemia, induced significant apoptosis. In accordance with the findings of Kametsu et al.(2003), we found that increased durations of transient ischemia paralleled with increased numbers of apoptotic cells in the ipsilateral hemisphere. We could not observe the same effect on the lesion volumes, because in the groups of 12.5- and 15-min ischemia number of animals with infarction was few ( 1 out 6 and 3 out of 7, respectively). Apoptotic cell death as a consequence of TIA-like brief transient focal brain ischemia evolves in a progressive and delayed manner (Du et al., 1996). Our results indicate that apoptosis induced by an episode of 10min ischemia is not aggravated from $24 \mathrm{~h}$ to 7 days after reperfusion. Similarly, MRI follow-up ensured that TIA induced with 10min MCAO is associated with no delayed MRI lesion up to 7 days. Additionally, total brain volumes on Day 1 and Day 7 were indifferent in this group of animals. Together with the lack of any other visible abnormalities in MRI, these data suggest that no early atrophy follows a TIA-level ischemic insult. Long-term follow-up studies incorporating versatile methodology are warranted for searching long-term cognitive consequences of TIA in rodents.

Preconditioning-ischemic tolerance paradigms used both surgical and intraluminal MCAO. With intraluminal method, three 10-min intervals of transient MCAO (separated with 45 min periods of reperfusion) produced no histologically proven brain injury at $24 \mathrm{~h}$, but a gross patchy infarction in the caudaputamen and selective neuronal necrosis in the cortex at $72 \mathrm{~h}$ was found (Chen et al., 1996). Others using a similar preconditioning protocol found no lesions with TTC-staining, neither at 24 nor at $72 \mathrm{~h}$ (Alkayed et al., 2002). Toyoda et al. (1997) induced 20 min of MCAO with simultaneous bilateral common carotid artery occlusion in the rat without observing any TTC-derived lesion $48 \mathrm{~h}$ later. A single 10-min period of MCAO (by surgical method) in spontaneously hypertensive rats produced no visible brain injury on HE-stained sections neither in short-term (day 1 and 2) nor in long-term (week 2 and 4), and no signs of apoptotic cell death was found (Barone et al., 1998). Following $15 \mathrm{~min}$ of MCAO at day 3, MRI did not reveal pathologic findings, but histopathologic examinations showed selective neuronal death in the striatum (Fujioka et al., 1999). Taken together, these data from ischemic tolerance studies suggest that histopathological outcome of brief transient MCAO (5-20 min) varies mainly depending on the animal strain and the method used for pathological assessment. TTC-staining is not precise enough to exclude small patchy infarcts as it depends on an enzymatic reaction which occurs independent of selective neuronal necrosis since the involved enzymes are as well located in glial cells and leukocytes in addition to neuronal cells. As well MRI fails to recognize selective neuronal necrosis induced by brief focal ischemia, as in our study and in others (Li et al., 2000). 
To conclude, a clinically relevant TIA model is achieved in Wistar rats, using intraluminal suture occlusion of the MCA for $10 \mathrm{~min}$ or shorter periods. Such model may be utilized to explore molecular level pathophysiological determinants and to develop therapies against delayed consequences of a TIA.

\section{Experimental procedures}

\subsection{Animals}

Adult male Wistar rats, weighing 250 to 450 g (Harlan Nederland, Horst, The Netherlands) were housed before surgery in groups of five in a temperature- and humidity-controlled environment in a $12 / 12 \mathrm{~h} \mathrm{light/dark}$ cycle with free access to standard rodent food and tap water. After surgery, environmental conditions were kept same, except that animals were housed individually. Body temperature was monitored by a rectal thermometer throughout the surgical procedures and imaging, and the animals were maintained normothermic via a heating blanket. All experiments were performed in conformity with the guidelines of the National Institutes of Health Guide for the Care and Use of Laboratory Animals (1996). The local Animal Research Committee approved the study protocol. Altogether 51 animals were eligible to be included to the analyses. Twenty-eight animals were excluded due to various failures including weak or no drop in LDF values following attempts of MCA occlusion, no recovery of LDF values following mechanical withdrawal of the suture occluder, or due to subarachnoid hemorrhage. We made all efforts to minimize the number of animals used and their suffering. All evaluations were done blindly to the experimental group allocation of the animals.

\subsection{Transient focal cerebral ischemia-animal groups}

We used injectional anesthesia by an intraperitoneal injection of ketamine hydrochloride $(50 \mathrm{mg} / \mathrm{kg}$, Ketalar, Parke-Davis, Detroit, MI) and a subcutaneous injection of medetomidine hydrochloride $(0.5 \mathrm{mg} / \mathrm{kg}$, Domitor, Orion, Espoo, Finland). To induce transient focal cerebral ischemia we used intraluminal suture MCAO model with a slight modification (Tatlisumak et al., 1998). Briefly, the right common carotid artery and the right external carotid artery were permanently ligated. A home-made suture occluder (a 4-0 nylon monofilament, with its tip rounded by heating and coated by silicone) was inserted through an arteriotomy below the carotid bifurcation and advanced rostrally into the internal carotid artery until a mild resistance was felt, indicating occlusion of the orifice of the right MCA. Reperfusion was achieved by withdrawing the occluder after varying durations of MCAO: $5,10,12.5,15,25$, and 30 min of transient ischemia was tested. Control group consisted of sham animals, which experienced the very same surgery, except MCAO. After collecting and analyzing the data from these groups, we studied an additional group of animals (7-day group), to which 10 min of transient ischemia was induced, and reperfusion period was extended to 1 week. In this group, we searched for any delayed appearance of MRI lesions by repeated imaging at $24 \mathrm{~h}, 72 \mathrm{~h}$, and 1 week. The final numbers of animals in each group are given in the Table 1.

\subsection{Model criteria}

\subsubsection{Criterion 1, demonstration of MCAO and reperfusion}

Laser-Doppler flowmetry (LDF, Oxy-Flow, Oxford Optronix, Oxford UK) guidance (Strbian et al., 2006) ensured firstly the success of MCAO, secondly of recanalization. Values of CBF at baseline, occlusion, and reperfusion (immediately, and at 10, 20, $30 \mathrm{~min}$, and
$24 \mathrm{~h}$ after reperfusion) were recorded. MCAO was interpreted as successful if CBF dropped to below 25\% of baseline level after occlusion, and reperfusion was found sufficient if postreperfusion value (latest at $30 \mathrm{~min}$ post-reperfusion) was a minimum $50 \%$ of baseline level. Animals were excluded in cases of inadequate MCAO or inadequate reperfusion, and when subarachnoid hemorrhage was suspected due to severe and sustained decreases of $\mathrm{CBF}$ after occlusion and during reperfusion.

4.3.2. Criterion 2, demonstration of normal neurological status at $24 \mathrm{~h}$

We applied a six-point scale of sensorimotor findings (Tatlisumak et al., 1998) as follows: $0=$ no deficit, $1=$ failure to fully extend the left forepaw, 2 = circling to the left, 3 = decreased resistance to lateral push, $4=$ no spontaneous walking with a depressed level of consciousness, and $5=$ dead. No deficit was the prerequisite for fulfilling Criterion 2. Animals were evaluated at the end of reperfusion period (i.e. at $24 \mathrm{~h}$; in the 7-day group additionally at $72 \mathrm{~h}$ and 7 days).

\subsubsection{Criterion 3, demonstration of the absence of visible infarction}

After neurological assessment, animals received anesthesia for MRI. We used a 4.7 Tesla scanner (PharmaScan, Bruker BioSpin, Ettlingen, Germany) with a $90-\mathrm{mm}$ shielded gradient capable of producing a maximum gradient amplitude of $300 \mathrm{mT} / \mathrm{m}$ with a $80-\mu$ s rise time. A linear birdcage RF coil with an inner diameter of $38 \mathrm{~mm}$ was used. Diffusion-weighted imaging (DWI) was applied by using a spin-echo echo-planar imaging (EPI) sequence with two b values of 0 and $1037 \mathrm{~s} / \mathrm{mm}^{2}$, and diffusion was measured in the read gradient direction $(\mathrm{TR} / \mathrm{TE}=3000 / 32 \mathrm{~ms}$, matrix size $=128 \times 128$, field-of-view $=40 \times 40 \mathrm{~mm}$ ). Afterwards, a T2weighed spin echo (rapid acquisition with relaxation enhancement, RARE $)$ sequence $\left(\mathrm{TR} / \mathrm{TE}_{\mathrm{eff}}=2500 / 80 \mathrm{~ms}\right.$, matrix size $=256 \times 192$, field-of-view $=40 \times 40 \mathrm{~mm})$ was run. Both DWI and $\mathrm{T} 2$-weighted sequences had seven 2 -mm-thick slices covering the entire brain. Images were evaluated visually by an investigator blind to animal groups to detect any region with increased signal indicating ischemic lesion. Ischemic lesions on T2-WI scans were manually outlined in each slice (Schneider et al., 2012). Lesion areas were summed-up, and multiplied by slice thickness yielding lesion volume.

\subsection{Tissue handling and histological evaluations}

After a lethal dose $(300 \mathrm{mg} / \mathrm{kg}$ ) of intraperitoneal pentobarbital (Mebunat, $60 \mathrm{mg} / \mathrm{mL}$, Orion, Espoo, Finland) injection, we performed cardiac perfusion-fixation with ice-cold saline. Brains were cut to 2-mm-thick slices and fixed in formaldehyde, and embedded in paraffin blocks. We cut 4- $\mu \mathrm{m}$ slices with a microtome (Leica SM2000 R, Leica Microsystems Nussloch GmbH, Nussloch, Germany) and HE or terminal deoxynucleotidyl transferase-mediated dUTPbiotin nick end-labeling (TUNEL, In Situ Cell Death Detection Kit, Fluorescein, Roche Diagnostics Oy, Espoo, Finland) stainings were applied (the latter according to the manufacturer's instructions). Stained slices were assessed under a light microscope (Axioplan2 Imaging, Carl Zeiss MicroImaging GmbH, Göttingen, Germany) by investigators who were blinded to the experimental groups.

In TUNEL-stained slices, we counted TUNEL-positive cells in both hemispheres, in three regions: the hippocampus, the frontoparietal cortex, and the caudaputamen. The ratio of the ipsilateral count to the contralateral count yielded the TUNEL-index.

In the HE-stained specimens, we searched for ischemic changes and necrotic findings in the hippocampus, caudaputamen, and frontoparietal cortex of both hemispheres (Pedrono et al., 2010). The pathology found in each area was scored as follows: $0=$ no change, $1=$ scattered neuronal changes, $2=$ selective neuronal 
necrosis (necrotic findings limited mainly to specific neuron populations), and $3=$ infarction (pannecrosis characterized as the loss of affinity for hematoxylin in all cell types). Afterwards, total scores were calculated by summing scores from all three areas of both hemispheres.

\subsection{Statistical analysis}

Statistics were performed using SigmaStat (version 3.11, Systat Software, Inc., Chicago, IL). All values, except for the neurological scores, were presented as mean \pm SD. One-way analysis of variance (ANOVA) followed by Holm-Sidak post hoc test compared parametric data (body weights, rectal temperatures, LDF values) in multiple groups. Nonparametric data (HE scores and nonnormally distributed TUNEL-index data) were analyzed using Mann-Whitney Rank Sum test for 2 group comparisons and for multiple group comparisons Kruskal-Wallis ANOVA on ranks test followed by Dunn's method or Tukey test. Follow-up values of parametric parameters (body weights, rectal temperatures, LDF values) are tested for variance with paired $t$-test or ANOVA for repeated measures. Spearman rank correlation tests assessed the correlation between the duration of ischemia and the number of apoptotic cells or HE scores. A two-sided $P$ value of $<0.05$ was considered statistically significant.

\section{Author contributions}

A.D.T., E.T., T.T., and E.P. conceived and designed the experiments. E.P. and A.D.T. performed the experiments. E.T. and A.D.T. performed the histological analyses. U.A-R. designed the magnetic resonance imaging sequences and acquired the imaging data. A.D. T. performed the data analysis and interpretation. A.D.T., E.T., and T.T. wrote the manuscript. All authors discussed the data and edited the manuscript. All authors approved the final version and agreed on submission.

\section{Disclosures}

None.

\section{Conflict of interest}

None.

\section{Acknowledgments}

This study was funded by the European Union (Grant No. FP7 202213), Finnish Academy of Sciences, and the Helsinki University Central Hospital.

\section{References}

Albers, G.W., Caplan, L.R., Easton, J.D., Fayad, P.B., Mohr, J.P., Saver, J.L., Sherman, D. G., Group, T.W., 2002. Transient ischemic attack-proposal for a new definition. N. Engl. J. Med. 347, 1713-1716.

Alkayed, N.J., Goyagi, T., Joh, H.D., Klaus, J., Harder, D.R., Traystman, R.J., Hurn, P.D., 2002. Neuroprotection and P450 2C11 upregulation after experimental transient ischemic attack. Stroke 33, 1677-1684.

Arsava, E.M., Gurer, G., Gursoy-Ozdemir, Y., Karatas, H., Dalkara, T., 2009. A new model of transient focal cerebral ischemia for inducing selective neuronal necrosis. Brain Res. Bull. 78, 226-231.

Bakker, F.C., Klijn, C.J., Jennekens-Schinkel, A., van der Tweel, I., Tulleken, C.A., Kappelle, L.J., 2003. Cognitive impairment in patients with carotid artery occlusion and ipsilateral transient ischemic attacks. J. Neurol. 250, 1340-1347.

Barone, F.C., White, R.F., Spera, P.A., Ellison, J., Currie, R.W., Wang, X., Feuerstein, G. Z., 1998. Ischemic preconditioning and brain tolerance: temporal histological and functional outcomes, protein synthesis requirement, and interleukin-1 receptor antagonist and early gene expression. Stroke 29, 1937-1950. discussion 1950-1931.

Chen, J., Graham, S.H., Zhu, R.L., Simon, R.P., 1996. Stress proteins and tolerance to focal cerebral ischemia. J. Cereb. Blood Flow Metab. 16, 566-577.

Du, C., Hu, R., Csernansky, C.A., Hsu, C.Y., Choi, D.W., 1996. Very delayed infarction after mild focal cerebral ischemia: a role for apoptosis? J. Cereb. Blood Flow Metab. 16, 195-201.

Easton, J.D., Saver, J.L., Albers, G.W., Alberts, M.J., Chaturvedi, S., Feldmann, E. Hatsukami, T.S., Higashida, R.T., Johnston, S.C., Kidwell, C.S., Lutsep, H.L., Miller, E., Sacco, R.L.Association AH, Council ASAS, Anesthesia CoCSa, Intervention CoCRa, Nursing CoC, Disease ICoPV, 2009. Definition and evaluation of transient ischemic attack: a scientific statement for healthcare professionals from the American Heart Association/American Stroke Association Stroke Council; Council on Cardiovascular Surgery and Anesthesia; Council on Cardiovascular Radiology and Intervention; Council on Cardiovascular Nursing; and the Interdisciplinary Council on Peripheral Vascular Disease. The American Academy of Neurology affirms the value of this statement as an educational tool for neurologists. Stroke 40, 2276-2293.

Ejaz, S., Emmrich, J.V., Sawiak, S.J., Williamson, D.J., Baron, J.C., 2015. Cortical selective neuronal loss, impaired behavior, and normal magnetic resonance imaging in a new rat model of true transient ischemic attacks. Stroke 46, 1084 1092.

Fink, K., Zhu, J., Namura, S., Shimizu-Sasamata, M., Endres, M., Ma, J., Dalkara, T. Yuan, J., Moskowitz, M.A., 1998. Prolonged therapeutic window for ischemic brain damage caused by delayed caspase activation. J. Cereb. Blood Flow Metab. 18, 1071-1076.

Fujioka, M., Taoka, T., Matsuo, Y., Hiramatsu, K.I., Sakaki, T., 1999. Novel brain ischemic change on MRI. Delayed ischemic hyperintensity on T1-weighted images and selective neuronal death in the caudoputamen of rats after brief focal ischemia. Stroke 30, 1043-1046.

Glantz, L., Avramovich, A., Trembovler, V., Gurvitz, V., Kohen, R., Eidelman, L.A. Shohami, E., 2005. Ischemic preconditioning increases antioxidants in the brain and peripheral organs after cerebral ischemia. Exp. Neurol. 192, 117-124.

Glazier, S.S., O'Rourke, D.M., Graham, D.I., Welsh, F.A., 1994. Induction of ischemic tolerance following brief focal ischemia in rat brain. J. Cereb. Blood Flow Metab. $14,545-553$

Hunter, A.J., Hatcher, J., Virley, D., Nelson, P., Irving, E., Hadingham, S.J., Parsons, A.A., 2000. Functional assessments in mice and rats after focal stroke. Neuropharmacology 39, 806-816.

Inatomi, Y., Kimura, K., Yonehara, T., Fujioka, S., Uchino, M., 2004. DW abnormalities and clinical characteristics in TIA patients. Neurology 62, 376380.

Kametsu, Y., Osuga, S., Hakim, A.M., 2003. Apoptosis occurs in the penumbra zone during short-duration focal ischemia in the rat. J. Cereb. Blood Flow Metab. 23, 416-422.

Lee, S.H., Kim, M., Kim, Y.J., Kim, Y.A., Chi, J.G., Roh, J.K., Yoon, B.W., 2002. Ischemic intensity influences the distribution of delayed infarction and apoptotic cell death following transient focal cerebral ischemia in rats. Brain Res. 956, 14-23.

Li, F., Han, S.S., Tatlisumak, T., Liu, K.F., Garcia, J.H., Sotak, C.H., Fisher, M., 1999 Reversal of acute apparent diffusion coefficient abnormalities and delayed neuronal death following transient focal cerebral ischemia in rats. Ann. Neurol. 46, 333-342.

Li, F., Liu, K.F., Silva, M.D., Omae, T., Sotak, C.H., Fenstermacher, J.D., Fisher, M., Hsu, C.Y., Lin, W., 2000. Transient and permanent resolution of ischemic lesions on diffusion-weighted imaging after brief periods of focal ischemia in rats: correlation with histopathology. Stroke 31, 946-954.

Naylor, M., Bowen, K.K., Sailor, K.A., Dempsey, R.J., Vemuganti, R., 2005 Preconditioning-induced ischemic tolerance stimulates growth factor expression and neurogenesis in adult rat hippocampus. Neurochem. Int. 47, $565-572$.

Oliff, H.S., Weber, E., Eilon, G., Marek, P., 1995. The role of strain/vendor differences on the outcome of focal ischemia induced by intraluminal middle cerebral artery occlusion in the rat. Brain Res. 675, 20-26.

Pedrono, E., Durukan, A., Strbian, D., Marinkovic, I., Shekhar, S., Pitkonen, M., AboRamadan, U., Tatlisumak, T., 2010. An optimized mouse model for transient ischemic attack. J. Neuropathol. Exp. Neurol. 69, 188-195.

Puisieux, F., Deplanque, D., Bulckaen, H., Maboudou, P., Gelé, P., Lhermitte, M. Lebuffe, G., Bordet, R., 2004. Brain ischemic preconditioning is abolished by antioxidant drugs but does not up-regulate superoxide dismutase and glutathion peroxidase. Brain Res. 1027, 30-37.

Schmid-Elsaesser, R., Zausinger, S., Hungerhuber, E., Baethmann, A., Reulen, H.J. 1998. A critical reevaluation of the intraluminal thread model of focal cerebral ischemia: evidence of inadvertent premature reperfusion and subarachnoid hemorrhage in rats by laser-Doppler flowmetry. Stroke 29 2162-2170.

Schneider, C.A., Rasband, W.S., Eliceiri, K.W., 2012. NIH Image to ImageJ: 25 years of image analysis. Nat. Methods 9, 671-675.

Strbian, D., Karjalainen-Lindsberg, M.L., Tatlisumak, T., Lindsberg, P.J., 2006. Cerebral mast cells regulate early ischemic brain swelling and neutrophil accumulation. J. Cereb. Blood Flow Metab. 26, 605-612.

Takahashi, P.Y., Dyrbye, L.N., Thomas, K.G., Cedeno, O.Q., North, F., Stroebel, R.J. DeJesus, R.S., Targonski, P.V., 2009. The association of transient ischemic attack symptoms with memory impairment among elderly participants of the Third US National Health and Nutrition Examination Survey. J. Geriatr. Psychiatry Neurol. 22, 46-51. 
Tatlisumak, T., 2015. Implication of the recent positive endovascular intervention trials for organizing acute stroke care: European perspective. Stroke 46, 14681473.

Tatlisumak, T., Takano, K., Carano, R.A., Miller, L.P., Foster, A.C., Fisher, M., 1998 Delayed treatment with an adenosine kinase inhibitor, GP683, attenuates infarct size in rats with temporary middle cerebral artery occlusion. Stroke 29 1952-1958.

Toyoda, T., Kassell, N.F., Lee, K.S., 1997. Induction of ischemic tolerance and antioxidant activity by brief focal ischemia. Neuroreport 8, 847-851.
Walters, R.J., Fox, N.C., Schott, J.M., Crum, W.R., Stevens, J.M., Rossor, M.N., Thomas, D.J., 2003. Transient ischaemic attacks are associated with increased rates of global cerebral atrophy. J. Neurol. Neurosurg. Psychiatry 74, 213-216.

Yoshida, M., Nakakimura, K., Cui, Y.J., Matsumoto, M., Sakabe, T., 2004. Adenosine A (1) receptor antagonist and mitochondrial ATP-sensitive potassium channe blocker attenuate the tolerance to focal cerebral ischemia in rats. J. Cereb. Blood Flow Metab. 24, 771-779. 